\title{
Collaborative Hypertension Case Management by Registered Nurses and Clinical Pharmacy Specialists within the Patient Aligned Care Teams (PACT) Model
}

\author{
Jessica L. O'Neill, Pharm. D', Tori L. Cunningham, Pharm. D², Wyndy L. Wiitala, Ph.D³, and \\ Emily P. Bartley, Pharm. ${ }^{7}$
}

'Department of Ambulatory Care, Veterans Affairs (VA) Ann Arbor Healthcare System, Ann Arbor, MI, USA; ${ }^{2}$ Department of Pharmacy Services, VA Eastern Kansas Healthcare System, Dwight D. Eisenhower VA Medical Center, Leavenworth, KS, USA; ${ }^{3}$ VA Center for Clinical Management Research, Ann Arbor VA Health Services Research and Development Center of Excellence, Ann Arbor, MI, USA.

\begin{abstract}
BACKGROUND: Clinical Pharmacy Specialists (CPSs) and Registered Nurses (RNs) are integrally involved in the Patient Aligned Care Teams (PACT) model, especially as physician extenders in the management of chronic disease states. CPSs may be an alternative to physicians as a supporting prescriber for RN case management (RNCM) of poorly controlled hypertension.
\end{abstract}

OBJECTIVE: To compare CPS-directed versus physician-directed RNCM for patients with poorly controlled hypertension.

DESIGN: Non-randomized, retrospective comparison of a natural experiment.

SETTING: A large Midwestern Veterans Affairs (VA) medical center.

INTERVENTION: Utilizing CPSs as alternatives to physicians for directing RNCM of poorly controlled hypertension.

PATIENTS: All 126 patients attended RNCM appointments for poorly controlled hypertension between 20 September 2011 and 31 October 2011 with either CPS or physician involvement in the clinical decision making. Patients were excluded if both a CPS and a physician were involved in the index visit, or they were enrolled in Home Based Primary Care, or if they displayed non-adherence to the plan.

MAIN MEASURES: All data were obtained from review of electronic medical records. Outcomes included whether a patient received medication intensification at the index visit, and as the main measure, blood pressures between the index and next consecutive visit.

KEY RESULTS: All patients had medication intensification. Patients receiving CPS-directed RNCM had greater decreases in systolic blood pressure compared to those receiving physician-directed RNCM (14 $13 \mathrm{mmHg}$ versus $10 \pm 11 \mathrm{mmHg} ; p=0.04)$. After adjusting for the time between visits, initial systolic blood pressure, and prior stroke, provider type was no longer significant $(p=0.24)$. Change in diastolic blood pressure and attainment of blood pressure $<140$ / $90 \mathrm{~mm} \mathrm{Hg}$ were similar between groups $(p=0.93$, $p=0.91$, respectively).

Published online April 9, 2014
CONCLUSIONS: CPS-directed and physician-directed RNCM for hypertension demonstrated similar blood pressure reduction. These results support the utilization of CPSs as prescribers to support RNCM for chronic diseases.

KEY WORDS: pharmacist; hypertension; patient centered medical home; patient aligned care teams; case management.

J Gen Intern Med 29(Suppl 2):S675-81

DOI: $10.1007 / \mathrm{s} 11606-014-2774-4$

(c) Society of General Internal Medicine 2014

\section{INTRODUCTION}

Cardiovascular disease is a common cause of disability and death in the United States and around the world. Hypertension, a major cardiovascular disease risk factor, affected $30.4 \%$ of the adult population of the United States between 2003 and 2010. ${ }^{1}$ Controlling hypertension decreases the risk of cardiovascular disease including myocardial infarction, stroke, heart failure, and death; however, over $50 \%$ of patients with hypertension do not have adequate control of their blood pressure. ${ }^{1}$ This often occurs despite access to healthcare and health insurance. ${ }^{1}$

The Community Preventive Services Task Force recommends team-based care to improve hypertension management in primary care clinics. ${ }^{2}$ Patient Aligned Care Teams (PACT) is the Veterans Affairs (VA) approach to implementing patient-centered, team-based primary care throughout the VA. ${ }^{3}$ One of the pillars of the PACT model is to increase access to care for patients via a coordinated team effort centered on the patient-primary care provider relationship. ${ }^{3}$ Services such as pharmacist and registered nurse case management (RNCM) are designed to support this goal of increased access to care for patients with chronic diseases such as hypertension. Although there are increasing data supporting registered nurse (RN) and pharmacist involvement in chronic disease care manage- 
ment, $^{4-12}$ little information is available on how to best integrate pharmacist capabilities with those of nurses in new patient-centered primary care models such as PACT.

One potential problem with RNCM is the need for additional support from a prescribing healthcare provider in the case of medication adjustments or renewals, laboratory orders, and referrals. Utilizing Clinical Pharmacy Specialists (CPSs) as prescribing professionals may enhance RNCM by reducing bottlenecks in the clinic flow driven by limited physician availability to RNs, which commonly occurs when RN case managers are involved in chronic disease state management. This model of RNCM, outlined in Fig. 1 and described in more detail below, is in place at one VA Medical Center.

In this facility, patients are referred to their assigned PACT $\mathrm{RN}$ for either face-to-face or telephone follow-up appointments for a variety of chronic disease states including hypertension. During visits for patients with poorly controlled hypertension, the RN assesses each patient independently. If hypertension remains poorly controlled or changes are indicated, the RN presents the case either to a physician or a CPS to collaboratively design a plan of care, which may include patient education on the self-management of their disease, changing medication therapy, consulting referral services, laboratory test ordering and/or arranging follow-up. The RN may chose to discuss the case with either a CPS (designated as "CPS-directed RNCM") or a physician (designated as "physician-directed RNCM"), based either on availability of the provider or complexity of the patient. The $\mathrm{RN}$ then returns to the patient and communicates any changes in the plan to the patient.

This study aims to assess these expanded CPS and RN roles, taking advantage of a naturalistic innovation described above. The primary objective was to compare blood pressure between CPS-directed and physician-directed RNCM. Comparable blood pressure changes between groups, regardless of prescriber type, would suggest that CPS-directed RNCM may be an acceptable alternative to physician-directed RNCM in PACT.

\section{Study Design}

\section{METHODS}

This study was a non-randomized, retrospective comparison of a natural experiment.

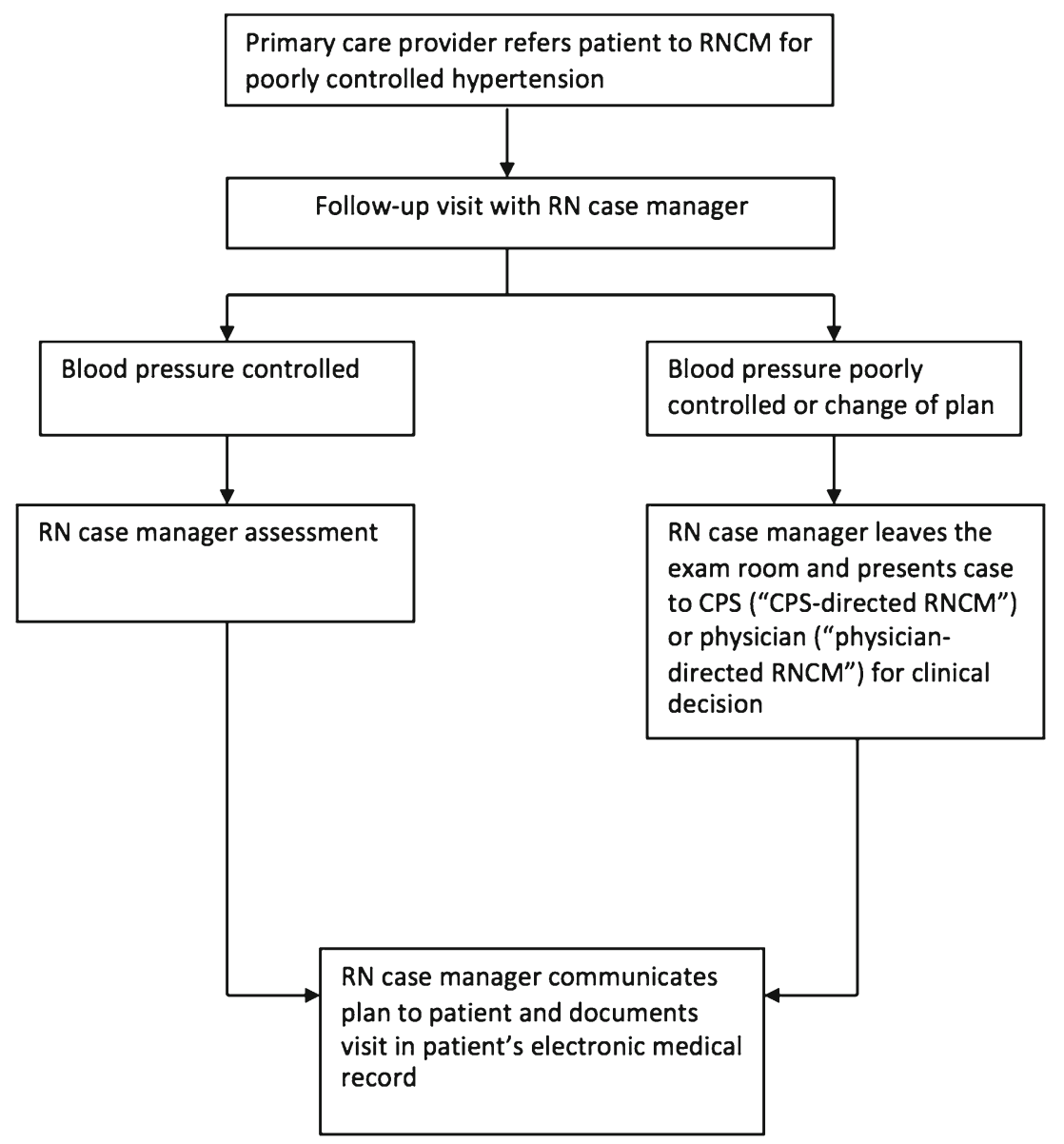

Figure 1. VA Ann Arbor Healthcare System (VAAAHS) model for RNCM of poorly controlled hypertension. 


\section{Sampling}

Institutional Review Board approval was achieved, then lists of patients with RNCM appointments from 1 May 2011 to 1 November 2011 were obtained via a report generated in VISTA (VHA Information Systems \& Technology Architecture) searching for the note title "Primary Care Nursing," which is utilized in RNCM encounters. Next, each patient's chart was reviewed against inclusion and exclusion criteria beginning with those who had appointments on 1 November 2011 and proceeding in reverse chronological order until 20 September 2011, when the pre-specified sample size of 63 patients per group was achieved. Patients were eligible for inclusion in the study if they had face-to-face or telephone appointments with an RN case manager for poorly controlled hypertension with either physician-directed or CPS-directed clinical decision making at the index encounter, as noted on the RN documentation of the clinic visit. Patients were excluded for any of the following reasons: neither a physician nor a CPS was listed as the designated provider on the index encounter note, both a physician and a CPS were involved in the same index RNCM encounter, the patient displayed non-adherence to treatment or follow-up, or the patient was enrolled in Home Based Primary Care. Provider type was determined by either an electronic signature from a CPS or a physician on the progress note associated with the RNCM visit, or a notation from the RN on the progress note indicating the prescribing provider. Nonadherence to treatment was determined if it was explicitly documented in the RNCM encounter or if no follow-up visit occurred after the index visit. Non-adherence to laboratory monitoring was determined by review of laboratory results after the index RNCM appointment. Included patients were placed into one of two study groups based on the type of provider collaborating with the RN during the visit: CPSdirected or physician-directed RNCM. Using a minimum average blood pressure change of $4 \mathrm{mmHg}(S D=8)$, alpha level of 0.05 , and $80 \%$ power, the required sample size was calculated to be 63 patients per group.

\section{Data Collection}

All data were collected by manual review of progress notes in the electronic medical record.

\section{Measures}

Demographic data collected included: age, sex, race, smoking status, body mass index and estimated renal function. ${ }^{13}$ The number of antihypertensive agents at index visit was collected, as well as comorbidities including diabetes, previous stroke or myocardial infarction, coronary artery disease, and dyslipidemia. Documentation from the index visit was reviewed to determine changes to the therapeutic plan recommended at the RNCM visit. To assess the main measure, blood pressure measurements at index and the next consecutive RNCM visit were collected for each patient. In the case of telephone encounters for patients who monitored their home blood pressure, the average blood pressure of multiple documented readings was used for the analysis. For face-to-face clinic appointments, home blood pressure cuff readings were included if it was documented that these were used instead of clinic blood pressure readings to determine progress to blood pressure goal, such as in the case of patients with "whitecoat hypertension." The following process measures were also collected: home blood pressure monitor prescriptions, time between visits, relevant laboratory tests, and referrals generated at the visit. Relevant laboratory monitoring was defined as a basic metabolic panel within 4 weeks of initiation or intensification of a diuretic, angiotensin converting enzyme inhibitor, angiotensin receptor blocker, or aldosterone antagonist.

\section{Statistical Analysis}

To assess the main measure, an independent samples $t$-test was conducted to compare difference in systolic blood pressure change between groups. An independent samples $t$-test was also conducted to compare diastolic blood pressure, as well as attainment of blood pressure less than 140/90 $\mathrm{mmHg}$, for patients using CPS-directed RNCM versus physician-directed RNCM.

Additional analyses examined the associations between the provider type and other patient measures including smoking status, comorbid conditions and referral services offered. Crosstab with Pearson's chi-square test was used to examine the relationships between categorical variables (sex, smoking status, race, comorbidities, renal function) and provider type. Fisher's exact test was used for associations where counts were less than five. A $t$-test was conducted to compare the number of blood pressure agents between groups. Separate preliminary regression models were conducted to examine the relationships between each of the patient measures with the outcomes (systolic blood pressure change, diastolic blood pressure change, attainment of blood pressure less than $140 / 90 \mathrm{mmHg}$ ), adjusting for baseline blood pressure measures. Measures that were either significant at $p<0.20$ or important for the current study (provider type, follow-up time, baseline blood pressure) were retained for inclusion in the multivariate model. All retained measures were simultaneously assessed using multiple regression models and were included in the final model if $p<0.05$. Rejected variables were re-added to the final model to confirm that they were not statistically significant or potential confounders. Provider type was the independent variable of interest, and therefore, was included in the final model regardless of significance level. Interactions between provider and patient measures were examined and included if $p<0.05$. All analyses used Stata version $12.1 .^{14}$ 
Table 1. Patient Characteristics

\begin{tabular}{|c|c|c|c|}
\hline & $\begin{array}{l}\text { CPS- } \\
\text { directed }\end{array}$ & $\begin{array}{l}\text { Physician- } \\
\text { directed }\end{array}$ & $\begin{array}{l}p \\
\text { value }\end{array}$ \\
\hline Patients per group & 63 & 63 & \\
\hline Male* & $61(96.8)$ & $62(98.4)$ & 1.00 \\
\hline Race* & & & 0.01 \\
\hline White & $37(58.7)$ & $52(82.5)$ & \\
\hline African American & $6(9.5)$ & $4(6.4)$ & \\
\hline Unknown & $20(31.8)$ & $7(11.1)$ & \\
\hline Age (years) $†$ & $63.4 \pm 9.8$ & $63.8 \pm 10.0$ & 0.82 \\
\hline Body mass index $\left(\mathrm{kg} / \mathrm{m}^{2}\right) \dagger$ & $32.5 \pm 6.6$ & $32.6 \pm 6.3$ & 0.91 \\
\hline Smoker* & $22(34.9)$ & $18(28.6)$ & 0.44 \\
\hline $\begin{array}{l}\text { Number of blood pressure } \\
\text { agents } \dagger\end{array}$ & $2.3 \pm 1.3$ & $2.2 \pm 1.2$ & 0.78 \\
\hline Diabetes mellitus* & $31(49.2)$ & $31(49.2)$ & 1.00 \\
\hline Heart failure* & $12(19.1)$ & $9(14.3)$ & 0.47 \\
\hline Previous stroke* & $8(12.7)$ & $4(6.4)$ & 0.36 \\
\hline Coronary artery disease* & $21(33.3)$ & $18(28.6)$ & 0.56 \\
\hline $\begin{array}{l}\text { Previous myocardial } \\
\text { infarction* }\end{array}$ & $6(9.5)$ & $6(9.5)$ & 1.00 \\
\hline Dyslipidemia* & $52(82.5)$ & $55(87.3)$ & 0.46 \\
\hline $\begin{array}{l}\text { Estimated glomerular } \\
\text { filtration rate } \\
\left(\mathrm{ml} / \mathrm{min} / 1.73 \mathrm{~m}^{2}\right)^{*}\end{array}$ & & & 1.00 \\
\hline$\geq 90$ & $10(15.9)$ & $10(15.9)$ & \\
\hline$\overline{6} 0-89$ & $36(57.1)$ & $37(58.7)$ & \\
\hline $30-59$ & $16(25.4)$ & $16(25.4)$ & \\
\hline $15-29$ & $1(1.6)$ & 0 & \\
\hline $\begin{array}{l}\text { Index systolic blood } \\
\text { pressure }(\mathrm{mm} \mathrm{Hg}) \dagger\end{array}$ & $149 \pm 12$ & $145 \pm 9$ & 0.03 \\
\hline $\begin{array}{l}\text { Index diastolic blood } \\
\text { pressure }(\mathrm{mm} \mathrm{Hg}) \dagger\end{array}$ & $78 \pm 12$ & $78 \pm 11$ & 0.94 \\
\hline $\begin{array}{l}\text { Index blood pressure }<140 / \\
90 \mathrm{mmHg}^{*}\end{array}$ & $9(14)$ & $13(21)$ & 0.35 \\
\hline $\begin{array}{l}\text { Blood pressure cuff offered } \\
\text { at index visit* }\end{array}$ & $3(4.8)$ & $5(7.9)$ & 0.72 \\
\hline $\begin{array}{l}\text { Average weeks between } \\
\text { index and follow-up visit } \dagger\end{array}$ & $3.4 \pm 1.9$ & $3.4 \pm 1.6$ & 0.92 \\
\hline $\begin{array}{l}\text { Appropriate laboratory } \\
\text { monitoring ordered* }\end{array}$ & 7 (18.9) & $14(35.9)$ & 0.13 \\
\hline \multicolumn{4}{|l|}{ Referrals offered: } \\
\hline Dietitian* & $2(3.2)$ & $6(9.5)$ & 0.27 \\
\hline Weight loss program* & $3(4.8)$ & $4(6.4)$ & 0.68 \\
\hline $\mathrm{CCHT}^{* *}$ & $2(3.2)$ & 0 & - \\
\hline
\end{tabular}

* Number of patients (percentage)

tMean $\pm S D$

${ }^{7}$ Care Coordination/Home Telehealth (CCHT) program uses video technology and messaging devices in the patient's home to communicate home blood pressure readings to an assigned $R N$ care coordinator at the VA facility. ${ }^{15}$

\section{RESULTS}

Out of 184 patients evaluated, 126 patients $(68.5 \%)$ were included: 63 each in the CPS-directed and physiciandirected groups. Fifty-eight patients $(31.5 \%)$ were excluded for the following reasons: $14(24 \%)$ due to CPS and physician involvement in the same index RNCM appointment, 21 (36\%) due to non-adherence to either laboratory monitoring orders or follow-up RNCM visits, and $23(40 \%)$ due to non-adherence to recommended change in therapy. Demographic characteristics were similar between groups except race $(p=0.01$; Table 1$)$. A greater proportion of patients in the physician-directed group had a race identified as "white." The majority of the sample across both groups was white (70.5\%); therefore, further analyses compared "white" versus "non-white." All patients had medication intensification at the index encounter.

Patients in the CPS-directed group had greater average decreases in systolic blood pressure compared to those in the physician-directed group, but the groups did not differ on measures of diastolic blood pressure change (see Table 2). There were fewer patients with blood pressure $<$ $140 / 90 \mathrm{mmHg}$ in the CPS group at index appointment $(n=$ $9,14 \%)$ compared to the physician group $(n=13,21 \%)$, although this difference was not significant $(p=0.35)$. At follow-up, the numbers of patients with blood pressure $<140 / 90 \mathrm{mmHg}$ were similar between the two groups $(n=$ $41,65 \%$ in the CPS group and $n=44,70 \%$ in the physician group, $p=0.57$ ).

Subsequent tests controlled for potential effects of patient measures (see Table 1). Each patient measure was evaluated in separate regression models that controlled for baseline value of blood pressure (see Table 3). Of the variables selected for continued testing for systolic blood pressure change (stroke, white); diastolic blood pressure change (age, number of blood pressure agents, diabetes, stroke, estimated renal function); and controlled blood pressure (age, number of blood pressure agents, diabetes, and stroke), only stroke was significant and remained in the final multivariate models for each outcome. Thus, the final multivariate regression models included provider type, follow-up time, index visit blood pressure, and prior stroke (see Table 4). Once these factors were controlled for, there was no significant effect for provider type on systolic blood pressure change $(p=0.24)$, diastolic blood pressure change ( $p=0.93$ ), or attaining blood pressure $<140 / 90 \mathrm{mmHg}$ ( $p=$ 0.91 ). Regardless of provider type, baseline measures for both systolic and diastolic blood pressure were significant predictors of change $(p=0.004$ and $p<0.001)$; higher initial values predicted greater reductions in blood pressure during the study period. Higher baseline systolic $(p=0.001)$ and

Table 2. Blood Pressure Change by Provider Type

\begin{tabular}{|c|c|c|c|c|c|c|}
\hline & \multicolumn{3}{|c|}{ Systolic blood pressure (mm Hg) } & \multicolumn{3}{|c|}{ "Diastolic blood pressure (mm Hg) } \\
\hline & CPS & Physician & $p$ & CPS & Physician & $p$ \\
\hline Index $($ Mean \pm SD $)$ & $149 \pm 12$ & $145 \pm 9$ & 0.03 & $78 \pm 12$ & $78 \pm 11$ & 0.94 \\
\hline Index range & $125-197$ & $120-164$ & & $55-102$ & $52-98$ & \\
\hline Follow-up $($ Mean $\pm \mathrm{SD})$ & $135 \pm 14$ & $135 \pm 11$ & 0.89 & $72 \pm 11$ & $73 \pm 11$ & 0.85 \\
\hline Follow-up range & $104-182$ & $102-172$ & & $52-105$ & $50-98$ & \\
\hline Difference between index and follow-up (Mean $\pm \mathrm{SD}$ ) & $14 \pm 13$ & $10 \pm 11$ & 0.04 & $6 \pm 10$ & $6 \pm 7$ & 0.90 \\
\hline
\end{tabular}


Table 3. Regression Models for Patient Measures on Blood Pressure Change, Adjusting for Blood Pressure at Index Visit

\begin{tabular}{|c|c|c|c|c|c|c|}
\hline & \multicolumn{2}{|c|}{$\begin{array}{l}\text { Systolic blood pressure } \\
\text { change* }\end{array}$} & \multicolumn{2}{|c|}{$\begin{array}{l}\text { Diastolic blood pressure } \\
\text { change* }\end{array}$} & \multicolumn{2}{|c|}{ Blood pressure $<140 / 90$} \\
\hline & $\beta(95 \%$ CI $)$ & $p$ & $\beta(95 \%$ CI $)$ & $p$ & OR (95 \% CI) & $p$ \\
\hline CPS-directed & $2.91(-1.14,6.97)$ & 0.16 & $0.26(-2.58,3.10)$ & 0.86 & $0.88(0.40,1.91)$ & 0.75 \\
\hline White & $3.81(-0.55,8.17)$ & 0.09 & $0.33(-2.80,3.45)$ & 0.84 & $1.09(0.47,2.52)$ & 0.84 \\
\hline Age (years) & $0.09(-0.12,0.30)$ & 0.40 & $0.12(-0.04,0.28)$ & 0.15 & $1.03(0.99,1.08)$ & 0.12 \\
\hline Body mass index $\left(\mathrm{kg} / \mathrm{m}^{2}\right)$ & $-0.07(-0.39,0.24)$ & 0.64 & $0.11(-0.11,0.33)$ & 0.32 & $0.99(0.93,1.05)$ & 0.68 \\
\hline Smoker & $0.38(-3.93,4.69)$ & 0.86 & $-0.74(-3.80,2.32)$ & 0.63 & $1.06(0.46,2.44)$ & 0.88 \\
\hline Number of blood pressure agents & $-0.14(-1.76,1.48)$ & 0.87 & $1.08(-0.16,2.32)$ & 0.09 & $1.28(0.92,1.78)$ & 0.14 \\
\hline Diabetes mellitus & $1.25(-2.76,5.26)$ & 0.54 & $2.06(-0.88,5.00)$ & 0.17 & $1.72(0.79,3.78)$ & 0.17 \\
\hline Heart failure & $-3.08(-8.44,2.28)$ & 0.26 & $-0.86(-4.76,3.04)$ & 0.66 & $0.83(0.29,2.39)$ & 0.74 \\
\hline Previous stroke & $9.68(2.96,16.39)$ & 0.01 & $5.33(0.59,10.08)$ & 0.03 & $3.52(0.73,16.98)$ & 0.12 \\
\hline Coronary artery disease & $1.81(-2.54,6.16)$ & 0.41 & $1.22(-1.93,4.36)$ & 0.45 & $1.35(0.58,3.19)$ & 0.49 \\
\hline Previous myocardial infarction & $1.33(-5.49,8.16)$ & 0.70 & $0.59(-4.28,5.46)$ & 0.81 & $1.56(0.38,6.35)$ & 0.53 \\
\hline Dyslipidemia & $0.81(-4.82,6.43)$ & 0.78 & $1.09(-2.91,5.09)$ & 0.59 & $1.38(0.47,4.05)$ & 0.56 \\
\hline Estimated glomerular filtration rate $\left(\mathrm{ml} / \mathrm{min} / 1.73 \mathrm{~m}^{2}\right)$ & $0.94(-2.11,4.00)$ & 0.54 & $1.52(-0.69,3.74)$ & 0.18 & $1.28(0.70,2.33)$ & 0.42 \\
\hline Average weeks between index and follow-up visit & $-0.12(-1.34,1.11)$ & 0.85 & $-0.40(-1.24,0.43)$ & 0.34 & $1.15(0.89,1.47)$ & 0.28 \\
\hline
\end{tabular}

*Change measures reflect difference calculated as (index - follow-up)

diastolic $(p=0.01)$ blood pressure was also associated with lower odds of attaining blood pressure $<140 / 90 \mathrm{mmHg}$. In addition, patients who had a prior stroke had greater reductions in both systolic $(p=0.007)$ and diastolic blood pressure $(p=0.04)$, as well as greater odds of attaining blood pressure $<140 / 90 \mathrm{mmHg}(p=0.05)$.

In terms of model performance, the final models for blood pressure change accounted for $14.6 \%$ of the total variance in systolic blood pressure change (adjusted $\mathrm{R}^{2}=$ 0.146 ) and $18.9 \%$ of the variance in diastolic blood pressure change (adjusted $\mathrm{R}^{2}=0.189$ ). The final model for attainment of blood pressure $<140 / 90 \mathrm{mmHg}$ accounted for $18.8 \%$ of the variance (pseudo $\mathrm{R}^{2}=0.188$ ). The model performed reasonably well for distinguishing between patients who attained blood pressure $<140 / 90 \mathrm{mmHg}$ versus those who did not $(\mathrm{AUC}=78.7 \%)$. Sensitivity was greater $(85.7 \%)$ than specificity (43.9\%), suggesting that the model performed better at correctly classifying patients who attained blood pressure $<140 / 90 \mathrm{mmHg}$ than correctly classifying patients who did not attain blood pressure $<140$ / $90 \mathrm{mmHg}$.

The process measure of laboratory monitoring was evaluated only in the subset of patients within each group for whom diuretics, angiotensin converting enzyme inhibitors, angiotensin receptor blockers or aldosterone antagonists were started or intensified at the index visit. Laboratory monitoring within 4 weeks was completed in 7 out of 37 possible cases in the CPS group (19\%) and 14 out of 39 possible cases in the physician group $(36 \% ; p=0.13)$. No significant associations between the remaining process measures and provider type were found (Table 1).

\section{DISCUSSION}

This study evaluated a novel model of care using CPSs with RNs collaboratively in patients with poorly controlled

Table 4. Multiple Regression Models to Predict Blood Pressure Change and Blood Pressure $<140 / 90 \mathrm{mmHg}$

\begin{tabular}{|c|c|c|c|c|}
\hline & $\beta$ or OR & $\mathbf{S E}$ & $p$ & $95 \% \mathrm{CI}$ \\
\hline \multicolumn{5}{|l|}{ Systolic blood pressure change* (adjusted $\mathrm{R}^{2}=0.146$ ) } \\
\hline CPS & 2.37 & 2.01 & 0.24 & $(-1.61,6.34)$ \\
\hline Weeks between index and follow-up RNCM visits & -0.11 & 0.60 & 0.85 & $(-1.3,1.08)$ \\
\hline Baseline systolic blood pressure & 0.30 & 0.10 & 0.004 & $(0.10,0.50)$ \\
\hline Prior stroke & 9.40 & 3.40 & 0.007 & $(2.67,16.12)$ \\
\hline \multicolumn{5}{|l|}{ Diastolic blood pressure change* (adjusted $\mathrm{R}^{2}=0.189$ ) } \\
\hline CPS & -0.13 & 1.43 & 0.93 & $(-2.97,2.71)$ \\
\hline Weeks between index and follow-up RNCM visits & -0.34 & 0.42 & 0.42 & $(-1.17,0.49)$ \\
\hline Baseline diastolic blood pressure & 0.34 & 0.06 & $<0.001$ & $(0.21,0.47)$ \\
\hline Prior stroke & 5.19 & 2.44 & 0.04 & $(0.36,10.02)$ \\
\hline \multicolumn{5}{|c|}{ Blood pressure $<140 / 90 \dagger\left(\right.$ pseudo $R^{2}=0.188$, AUROC $\left.=0.787\right)$} \\
\hline CPS & 0.95 & 0.42 & 0.91 & $(0.40,2.26)$ \\
\hline Weeks between index and follow-up RNCM visits & 1.10 & 0.16 & 0.52 & $(0.83,1.45)$ \\
\hline Baseline systolic blood pressure & 0.91 & 0.02 & 0.001 & $(0.86,0.96)$ \\
\hline Baseline diastolic blood pressure & 0.95 & 0.02 & 0.01 & $(0.91,0.99)$ \\
\hline Prior stroke & 7.12 & 6.99 & 0.05 & $(1.04,48.75)$ \\
\hline
\end{tabular}

*Change measures reflect difference calculated as (index - follow-up)

thogistic regression 
hypertension within the PACT model. Similar and clinically significant blood pressure reductions occurred in both the CPS-directed and physician-directed RNCM groups. Our results suggest that CPS-directed RNCM achieves blood pressure reduction comparable to physician-directed RNCM. Baseline systolic and diastolic blood pressure were predictors of systolic and diastolic blood pressure change, regardless of CPS-directed or physician-directed RNCM. Since patients were only included in the study if they had hypertension, this observation may be due to regression to the mean. Our study adds support to the inclusion of CPSs as an alternative to physicians for directing $\mathrm{RN}$ case managers who are involved in visits that require medication adjustments for patients with poorly controlled hypertension in the PACT model.

This study had important limitations. First, the study is a retrospective evaluation of a naturalistic innovation and thus is exploratory; this study is not designed to fully address effectiveness. Second, the VA context, including its primarily male patient population, may limit generalizability. Thirdly, due to the naturalistic design and retrospective nature, we were limited to previously documented, unstandardized data from a variety of clinicians. Therefore, we were unable to control for additional variables that could affect the primary outcome measure, such as other medications that affect blood pressure, patient-specific blood pressure goals, or complementary and alternative medicine. In addition, we could not control for all variables that might lead an RN to consult a physician over a CPS or vice versa. Finally, this study was not designed to assess safety and adherence of medications, or safety of and adherence to, this model.

Patients who were non-adherent with treatment or followup recommendations were excluded from the data analysis. Given that the RN was directly providing the care and the discussion of the case occurred away from the patient in this model, adherence differences based on the type of provider that the RN was collaborating with were not expected. Nonetheless, if there were adherence differences between groups, this may affect the primary outcome, and therefore it is not clear if the results would hold for patients exhibiting non-adherence with medications or follow-up instructions. Excluding these patients could have also affected the secondary measure reflecting appropriate laboratory monitoring, which appeared to have a low rate in both groups, but was more pronounced in the CPS group. This was an unexpected finding, but it remains unclear whether or not this accurately reflects laboratory monitoring ordered at the index RNCM visit, because the proportion of patients excluded from each group for non-adherence to laboratory monitoring recommendations was not collected.

In our study, patients receiving collaborative team-based RNCM for hypertension achieved clinically significant blood pressure reductions whether the prescribing provider was a CPS or a physician. CPS-directed RNCM may increase patients' access to care, not only by adding a new service available to patients, but it may increase physicians' availability for other patient care activities, as CPSs function at a higher level with independent prescribing privileges within an expanded scope of clinical practice. These results support an expanded role for CPS-RN teams within the PACT model. CPSs may be an acceptable alternative to physicians as supporting prescribers for RNCM of poorly controlled hypertension.

Acknowledgements: We would like to thank our PACT CPS, RN, and physician colleagues and supervisors at the VA Ann Arbor Healthcare System Primary Care Clinic. In addition Denise Ramsey, MSN, RN and Wendy Morrish, MSN, RN provided assistance with an earlier draft of this article.

This model was previously presented prior to data collection under the title "Effect of clinical pharmacy specialist-staffed nurse case management on blood pressure" at the 2012 Great Lakes Pharmacy Resident Conference, Purdue University, West Lafayette, IN on 24 April 2012.

This study was presented as a poster titled "Collaborative hypertension case management by clinical pharmacy specialists and registered nurses in a VA primary care clinic," at the 2012 American College of Clinical Pharmacy Annual Meeting, Westin Diplomat Resort, Hollywood, FL on 23 October 2012.

The contents do not represent the views of the Department of Veterans Affairs or the United States government.

Conflict of Interest: The authors declare that they do not have a conflict of interest.

Financial Disclosures: None.

Corresponding Author: Jessica L. O'Neill, Pharm.D; Department of Ambulatory CareVeterans Affairs (VA) Ann Arbor Healthcare System, 2215 Fuller Road, Ann Arbor, MI 48105, USA (e-mail: Jessica.oneill@va.gov).

\section{REFERENCES}

1. Centers for Disease Control and Prevention. Vital Signs: Awareness and treatment of uncontrolled hypertension among adults-United States, 20032010. Morbidity and Mortality World Report, September 7 2012;61(35): 7039. Available from: www.cdc.gov/mmwr/preview/mmwrhtml/ mm6135a3.htm?s_cid=mm6135a3 w. Accessed January 2, 2014.

2. The Guide to Community Preventative Services. Cardiovascular Disease Prevention and Control: Team-Based Care to Improve Blood Pressure control. Available from: www.thecommunityguide.org/cvd/ teambasedcare.html. Accessed January 2, 2014.

3. United States Department of Veterans Affairs. Patient Aligned Care Team (PACT). Available from: http://www.va.gov/primarycare/pcmh/. Accessed January 2, 2014

4. Centers for Disease Control and Prevention. A program guide for public health: Partnering with pharmacists in the prevention and control of chronic diseases. Available from: http://www.cdc.gov/dhdsp/programs/ nhdsp_program/docs/pharmacist_guide.pdf. Accessed January 2, 2014.

5. McCord AD. Clinical impact of a pharmacist-managed diabetes mellitus drug therapy management service. Pharmacotherapy. 2006;26(2):248253.

6. Choe HM, Mitrovich S, Dubay D, Hayward RA, Krein SL, Vijan S. Proactive case management of high-risk patients with type 2 diabetes mellitus by a clinical pharmacist: a randomized controlled trial. Am J Manage Care. 2005;11:253-260. 
7. Fahey T, Schroeder K, Ebrahim S. Educational and organizational interventions used to improve the management of hypertension in primary care: a systematic review. Br J Gen Pract. 2005;55(520):875-882.

8. Green BB, Cook AJ, Ralston JD, et al. Effectiveness of home blood pressure monitoring, web communication, and pharmacist care on hypertension control: a randomized controlled trial. JAMA. 2008;299(24):2857-2867.

9. Bodenheimer $\mathbf{T}$, Wagner $\mathbf{E H}, \mathbf{G r u m b a c h ~} \mathbf{K}$. Improving primary care for patients with chronic illness: the chronic care model, part 2. JAMA. 2002;288(15):1909-1914.

10. Ireland S, MacKenzie G, Gould L, Dassinger D, Koper A, LeBlanc K. Nurse case management to improve risk reduction outcomes in a stroke prevention clinic. Can J Neurosci Nurs. 2010;32(4):7-13.

11. Ishani A, Cole $\mathbf{P}$, Greer $\mathbf{N}$, et al. Effect of nurse case management compared with usual care on controlling cardiovascular risk factors in patients with diabetes. Diabetes Care. 2011;34:16891694.

12. Welch G, Garb J, Zagarins S, Lendel I, Gabbay RA. Nurse diabetes case management interventions and blood glucose control: results of a metaanalysis. Diabetes Res Clin Pract. 2010;88:1-6.

13. Levey AS, Coresh J, Greene T, Stevens LA, Zhang Y, Hendriksen S. Using standardized serum creatinine values in the modification of diet in renal disease study equation for estimating glomerular filtration rate. Ann Intern Med. 2006; 145:247-254.

14. StataCorp. Stata Statistical Software: release 12. College Station: StataCorp LP; 2011.

15. United States Department of Veterans Affairs. Care Coordination/Home Telehealth. Available from: http://www.telehealth.va.gov/ccht/ index.asp. Accessed January 2, 2014. 